\title{
The feasibility and safety of disposable endoscope vs. conventional endoscope for upper gastrointestinal tract examination: a multicenter, randomized, parallel, non-inferiority trial
}

\section{Die Durchführbarkeit und Sicherheit von Einweg-Endoskopen bei der Untersuchung des oberen Gastrointestinaltrakts im Vergleich zu konventionellen Endoskopen: Eine multizentrische, randomisierte, parallele, Nichtunterlegenheits-Studie}

\section{(ㄷ) (ㄱ) $९$}

Authors

De-feng Li ${ }^{1}$, Rui-yue Shi ${ }^{1}$, Yan-hui Tian ${ }^{1}$, Zheng-lei Xu' ${ }^{1}$, Ying-sheng Zhou ${ }^{2}$, Xian-jiu Sun ${ }^{2}$, Jin-wei Cai ${ }^{2}$, Yuan-yuan Fang ${ }^{2}$, Huan Peng' ${ }^{2}$, Jia-min Wang ${ }^{2}$, Tao Dong ${ }^{3}$, Yong-de Cai ${ }^{3}$, Jun Yao ${ }^{1}$, Li-sheng wang ${ }^{1}$

\section{Affiliations}

1 Department of Gastroenterology, Shenzhen People's Hospital (The Second Clinical Medical College, Jinan University, The First Affiliated Hospital, Southern University of Science and Technology)

2 Department of Gastroenterology, University of Chinese Academy of Sciences Affiliated Shenzhen Hospital

3 Shenzhen Pengrui Intelligent Image Co., Ltd

Schlüsselwörter neuartiges Einweg-Endoskop, Durchführbarkeit, Sicherheit

Key words

novel disposable endoscope, feasibility, safety

received 23.01.2021

accepted 08.07.2021

published online 12.11.2021

\section{Bibliography}

Z Gastroenterol 2022; 60: 1314-1319

DOI $10.1055 / a-1555-0568$

ISSN $0044-2771$

\section{(c) 2021. The Author(s).}

This is an open access article published by Thieme under the terms of the Creative Commons Attribution-NonDerivative-NonCommercial-License, permitting copying and reproduction so long as the original work is given appropriate credit. Contents may not be used for commercial purposes, or adapted, remixed, transformed or built upon. (https://creativecommons.org/licenses/by-nc-nd/4.0/).

Georg Thieme Verlag KG, Rüdigerstraße 14,

70469 Stuttgart, Germany

Correspondence

Li-sheng wang

The First Affiliated Hospital, Southern University of Science and Technology)

Department of Gastroenterology, Shenzhen People's Hospital (The Second Clinical Medical College, Jinan University wanglsszrmyy@163.com
Additional material is available at https://doi.org/ 10.1055/a-1555-0568.

\section{ABSTRACT}

Background A disposable upper gastrointestinal endoscope can effectively decrease infectious outbreaks associated with endoscope reuse. In the present study, we aimed to evaluate the feasibility and safety of a disposable endoscope for upper gastrointestinal examination.

Methods In a prospective, randomized trial, 144 upper endoscopic procedures were allocated to either the disposable endoscope group or the conventional endoscope group. The primary outcomes were rates of excellent and good image qualities and maneuverability satisfaction. The second outcome included procedure duration, endoscopic diagnosis, and adverse events.

Results A total of 144 subjects were enrolled in the present analysis and prospectively randomized to 2 study groups. Finally, 70 and 69 subjects were enrolled in the novel disposable endoscope group and the conventional endoscope group, respectively, due to the schedule cancellation of 5 subjects. The baseline characteristics of the patients were similar in both groups. The excellent and good image quality rates and maneuverability satisfaction of the novel disposable endoscope were not inferior to the conventional endoscope $(p=0.99$ and $p=0.99$, respectively). Moreover, no significant between-group difference was observed in the endoscopic results and adverse events ( $p=0.30$ and $p=1$, respectively). However, the procedure duration in the novel disposable endoscope was longer compared with the conventional endoscope $(8.40 \pm 4.28$ min vs. $5.12 \pm 2.65 \mathrm{~min}, \mathrm{p}<0.001)$.

Conclusions The novel disposable endoscope was as safe, effective, and maneuverable as a conventional endoscope. However, the novel disposable endoscope was associated with a longer procedure duration. 


\section{ZUSAMMENFASSUNG}

Hintergrund Einweg-Endoskope für den oberen Gastrointestinaltrakt können Infektionsausbrüche, die auf die Wiederverwendung von Endoskopen zurückzuführen sind, wirksam verringern. In der vorliegenden Studie sollte die Durchführbarkeit und Sicherheit von Einweg-Endoskopen bei der Untersuchung des oberen Gastrointestinaltrakts bewertet werden.

Methoden In einer prospektiven, randomisierten Studie wurden 144 endoskopische Eingriffe des oberen Gastrointestinaltrakts entweder der Einweg-Endoskop-Gruppe oder der konventionellen Endoskop-Gruppe zugewiesen. Die primären Endpunkte waren die Rate der guten Bildqualität sowie die Zufriedenheit mit der Manövrierfähigkeit. Zu den zweiten Endpunkten gehörten die Dauer des Eingriffs, die endoskopische Diagnose und unerwünschte Ereignisse.

Ergebnisse Insgesamt wurden 144 Probanden in die vorliegende Studie aufgenommen und prospektiv auf zwei Studien- gruppen randomisiert. Da fünf Probanden ausfielen, wurden schließlich 70 Probanden in die Einweg-Endoskop-Gruppe und 69 in die konventionelle Endoskop-Gruppe eingeschlossen. Die Patienten beider Gruppen zeigten ähnliche Ausgangscharakteristika. Die Bildqualität und die Zufriedenheit mit der Manövrierfähigkeit des neuartigen Einweg-Endoskops waren nicht schlechter als bei einem konventionellen Endoskop ( $P=0,99$ bzw. $P=0,99)$. Außerdem wurde bei den endoskopischen Ergebnissen und unerwünschten Ereignissen kein signifikanter Unterschied zwischen den Gruppen beobachtet $(P=0,30$ bzw. $P=1)$. Allerdings dauerte der Eingriff mit dem neuartigen Einweg-Endoskop länger als mit dem konventionellen Endoskop $(8,40 \pm 4,28 \mathrm{~min}$ vs. 5,12 $\pm 2,65 \mathrm{~min}, \mathrm{P}$ $<0,001$ ).

Schlussfolgerungen Das neuartige Einweg-Endoskop war genauso sicher, effektiv und manövrierfähig wie das konventionelle Endoskop. Allerdings war das neuartige Einweg-Endoskop mit einer längeren Verfahrensdauer verbunden.

\section{ABBREVIATIONS}

COVID-19 coronavirus disease-19

ERCP endoscopic retrograde cholangiopancreatography

\section{Introduction}

Gastrointestinal endoscopes are widely contaminated with patients' native flora, and the incidence of infectious transmission is reported to be 3.7 and 1.6 per 1,000 gastrointestinal endoscopic procedures [1, 2]. Therefore, it is necessary to prevent endoscope-related infections during subsequent uses. However, it is estimated that the disinfection failure rate is nearly $2.0 \%$ despite stringent reprocessing [3, 4]. Importantly, persistent endoscope contamination can result in device-related infections. In particular, the COVID-19 outbreak has recently spread throughout the world and is mainly transmitted through direct contact $[5,6]$. Endoscopic procedures can increase the risk of COVID-19 transmission to endoscopists, assistants, and patients potentially also by contaminated endoscopes [7, 8].

Effective strategies to eradicate endoscope-related contamination remain elusive because the reasons for endoscope-related contamination are complicated, mainly including reprocessing lapses, endoscope defects, etc. [9]. However, Muthusamy et al. have reported that a disposable duodenoscope can effectively decrease infectious transmission risk associated with the reuse of a duodenoscope during an endoscopic retrograde cholangiopancreatography (ERCP) procedure [10]. In the present study, we aimed to assess a new disposable upper gastrointestinal endoscope and compare the feasibility and safety of this new device with the conventional endoscope.

\section{Methods and Materials}

\section{Study design}

This was a multicenter, randomized, parallel, non-inferiority trial, and patients were recruited from 2 hospitals in China between December 2019 and June 2020. The study was approved by the Human Ethics Committees, and our research protocols complied with the Declaration of Helsinki. The study was registered online at http://www.chictr.org.cn (No. ChiCTR2000029945). Written informed consent was obtained from all subjects before their enrolment.

\section{Novel disposable upper gastrointestinal endoscope}

The novel disposable upper gastrointestinal endoscope (PR-IPD002) was made by Shenzhen PengRui Intelligent Image Co., Ltd., and consists of a computer monitor, image processor, control body, and endoscope. The details are available in Appendix 1.

\section{Patients}

The inclusion criteria were set as follows: (1) patients aged between 18 and 75 years; (2) patients scheduled for upper gastrointestinal endoscopy, and (3) patients who provided informed consent.

Patients with the following conditions were excluded from the present study: (1) severe cardiopulmonary disease, (2) gastrointestinal perforation, (3) shock, (4) severe laryngeal disease, (5) acute erosive esophagitis (such as misuse of strong acid or strong bases), (6) thoracoabdominal aortic aneurysm, (7) history of mental illness or cognitive impairment, (8) allergic to anesthetic drugs, (9) coagulation dysfunction, (10) hemoglobin <50 g/L, (11) pregnancy or lactation, and (12) participation in other clinical trials. 


\section{Endoscopists}

All endoscopists were adequately trained for the use of the novel disposable upper gastrointestinal endoscope and the image quality grading system at the initiation of this study (Supplement Table S1). Many multiple upper gastrointestinal images were independently reviewed and scored by each endoscopist. All results were summarized, and discrepancies were discussed and resolved through consensus. All procedures were performed by experienced endoscopists who had performed more than 10,000 upper gastrointestinal endoscopies.

\section{Randomization}

Routine laboratory tests results of patients, including electrocardiogram, blood counts, HBV, HIV, human chorionic gonadotrophin, and COVID-19 real-time PCR, were within the normal ranges. The patients were randomly allocated to either the disposable endoscope group or the conventional endoscope group. The randomization numbers were generated using a computer random number generator and placed in sealed opaque envelopes, which were opened at the beginning of the upper gastrointestinal endoscopy. However, due to the nature of this study, it was impossible to blind endoscopists and participants to the randomized allocations.

\section{Upper gastrointestinal endoscope procedure}

All patients fasted for $8 \mathrm{~h}$ before the procedure, and a mixture of $100 \mathrm{~mL}$ water, 20,000 U pronase (Beijing Tide Pharmaceutical Company, Beijing, China), $1 \mathrm{~g}$ sodium bicarbonate (Beijing Tide Pharmaceutical Company, Beijing, China), and $10 \mathrm{~mL}$ simethicone (Berlin-Chemie AG, Berlin, Germany) was given to the patients before the procedure. All patients received anesthesia through intravenous injection of $5 \mathrm{mg}$ midazolam and $50 \mathrm{mg}$ pethidine.

Both the novel disposable endoscopy and conventional endoscopy were performed by experienced endoscopists from Shenzhen People's Hospital and the University of Chinese Academy of Sciences Affiliated Shenzhen Hospital. A GIF-260 (Olympus, Japan) was used for the conventional endoscopy.

\section{Definition}

Comprehensive evaluation standards of image quality included observation, preservation, and analysis of digital image quality. The detailed evaluation contents are listed in Table S1.

Comprehensive evaluation standards for operability evaluation included operation satisfaction, visual fatigue, and physical fatigue. The detailed evaluation contents are listed in Table S2.

Procedure duration was defined as the time elapsed from the entry of the endoscope to the removal of the endoscope. Adverse

- Table 1 Baseline characteristics and outcomes.

\begin{tabular}{|c|c|c|c|}
\hline & Novel disposable endoscope & Conventional Endoscope & p-value \\
\hline \multicolumn{4}{|l|}{ Gender } \\
\hline - Male & 24 & 27 & \multirow[t]{2}{*}{0.55} \\
\hline - Female & 46 & 42 & \\
\hline Age & $43.8 \pm 10.8$ & $43.4 \pm 10.3$ & 0.71 \\
\hline \multicolumn{4}{|l|}{ Indications } \\
\hline - Abdominal pain & 23 & 24 & \multirow[t]{4}{*}{0.99} \\
\hline - Abdominal distention & 25 & 23 & \\
\hline - Screening & 15 & 16 & \\
\hline - Others & 7 & 6 & \\
\hline Procedure duration & $8.40 \pm 4.28$ & $5.12 \pm 2.65$ & $<0.001$ \\
\hline \multicolumn{4}{|l|}{ Endoscopic results } \\
\hline - Duodenal ulcer & 3 & 4 & \multirow[t]{8}{*}{0.30} \\
\hline - Gastric ulcer & 1 & 1 & \\
\hline - Reflux esophagitis & 4 & 1 & \\
\hline - Erosive gastritis & 52 & 54 & \\
\hline - Duodenal polyp & 1 & 1 & \\
\hline - Esophageal SMT & 3 & 0 & \\
\hline - Gastric SMT & 1 & 0 & \\
\hline - Gastric polyp & 5 & 8 & \\
\hline Adverse events & 0 & 0 & 1 \\
\hline
\end{tabular}


events included throat injury, Mallory-Weiss syndrome, bleeding, and perforation during 1-day follow-up.

\section{Outcomes}

The primary outcomes were the rates of excellent and good image qualities. The secondary outcomes included assessment of maneuverability, procedure duration, endoscopic detection, and adverse events.

\section{Statistical analysis}

The following formula was used to calculate the sample size.

$$
n_{T}=n_{c}=\frac{\left(Z_{1-\alpha / 2}+Z_{1-\beta}\right)^{2}\left[P_{c}\left(1-P_{c}\right)+P_{T}\left(1-P_{T}\right)\right]}{(|D|-\Delta)^{2}}
$$

$P_{T}$ indicates that the predicted excellent and good rates of image quality in the novel endoscope group were $96 \%$. Pc indicates that the predicted excellent and good rates of image quality in the conventional group were $96 \% .|D|=\left|P_{T}-P c\right| . \Delta$ was the non-inferiority margin defined as -0.1 . Seventy-two cases were needed in each group according to an alpha of 0.05 , a power of $10 \%$, and a dropout rate of $15 \%$.

All analyses were calculated using SPSS 23.0 software package (SPSS Company, Chicago, IL, USA). Categorical variables were expressed as frequencies (percentage), while continuous variables were expressed as mean \pm standard deviation or median (interquartile range) due to the distribution. Between-group differences in categorical variables were analyzed using the Chi-squared test or Fisher's exact test, and continuous variables were analyzed using the Student's t-test or a Mann-Whitney test.

\section{Results}

\section{Baseline characteristics}

A total of 144 eligible subjects were enrolled in the present study and randomly allocated to the 2 study groups. However, 2 subjects in the novel disposable endoscope group and 3 subjects in the conventional endoscope group were excluded because of the schedule cancellation. Finally, there were 70 and 69 subjects in

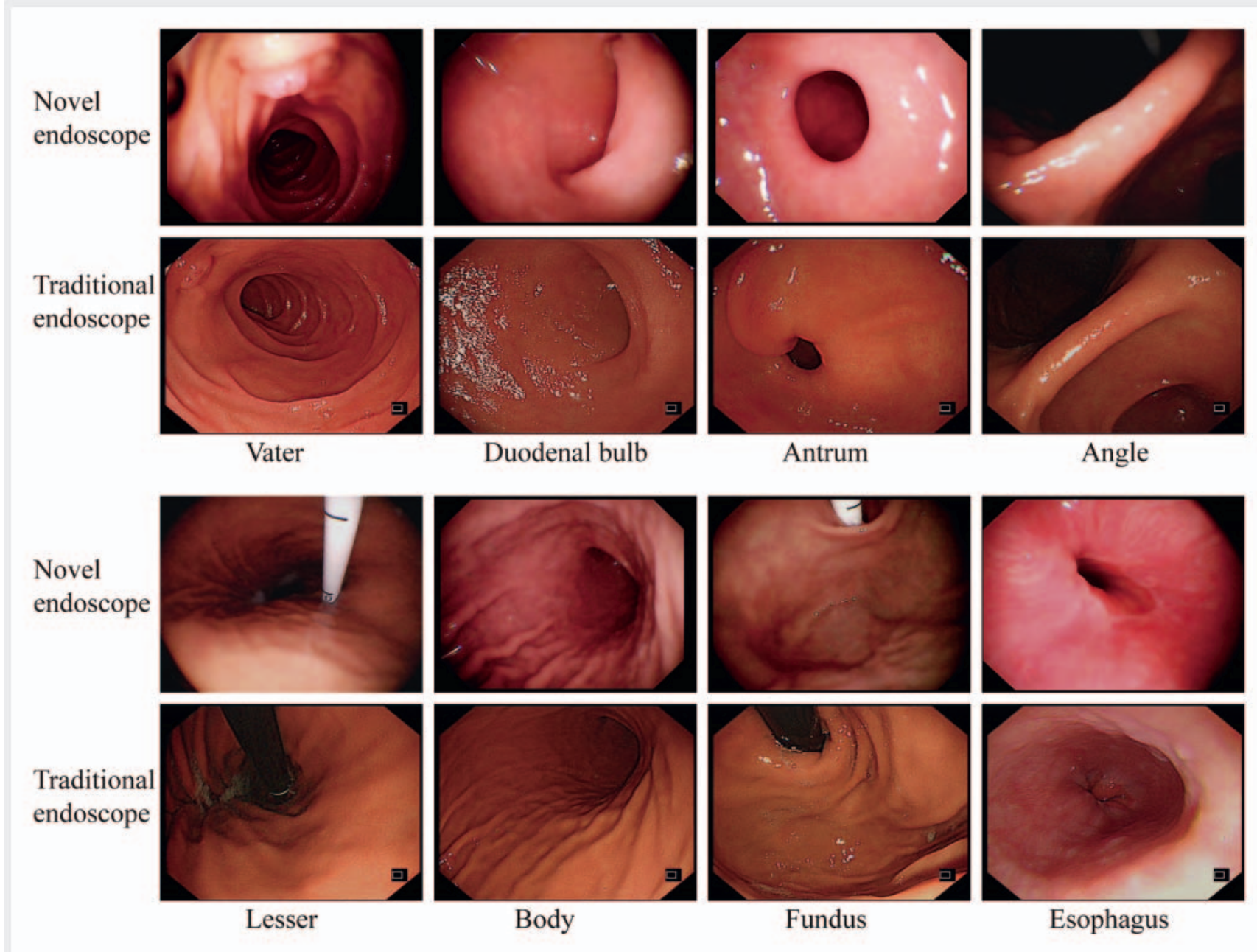

- Fig. 1 Endoscopic images of the same anatomical regions of the upper gastrointestinal tract between the novel disposable endoscope and traditional endoscope. 
the novel disposable endoscope group and the conventional endoscope group, respectively. There was no further dropout or loss to follow-up in both groups.

- Table 1 shows that there was no significant difference in terms of gender and age between the 2 groups ( $p=0.55$ and $p=0.71$, respectively). The indications for upper gastrointestinal endoscopic examination were similar between the 2 groups $(p=0.99)$. Moreover, no significant between-group difference was observed in the endoscopic results $(p=0.30)$. Indeed, there were no adverse events in either group $(p=1)$. However, the procedure duration in the novel disposable endoscope group was longer compared with the conventional endoscope group (8.40 \pm 4.28 min vs. $5.12 \pm 2.65$ min, $\mathrm{p}<0.001$ ) ( Table 1 ).

\section{Image quality and maneuverability assessment}

- Fig. 1 shows endoscopic images at the same anatomical regions of the upper gastrointestinal tract in both groups. Fig. 2A shows that the rates of excellent and good image qualities were similar between the 2 groups (98\% vs. $100 \%, p=0.99$ ). Upper gastrointestinal tract examination was successfully accomplished in both groups, and the maneuverability satisfaction of the novel disposable endoscope was not inferior to the conventional endoscope (98\% vs. $100 \%, p=0.99)$ ( $\triangleright$ Fig. 2 B).

\section{Discussion}

In the present study, we, for the first time, compared the feasibility and safety of a novel disposable endoscope and a conventional endoscope for upper gastrointestinal tract examination. We found that the image quality, maneuverability satisfaction, endoscopic diagnosis, and adverse events were comparable between the 2 approaches. However, the procedure duration for the novel dispo-

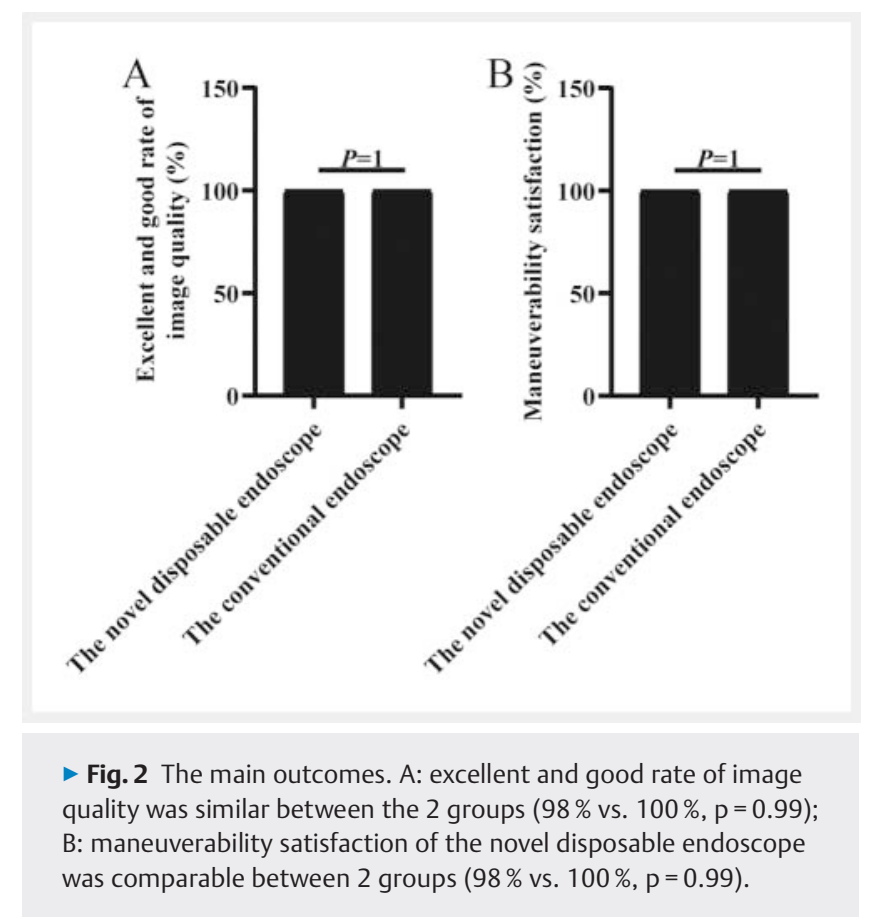

sable endoscope was longer compared with the conventional endoscope.

Previous studies have reported carbapenemase-producing Klebsiella pneumonia outbreaks in hospitals because of contaminated reusable duodenoscopes during ERCP procedures [11, 12]. Duodenoscopes have been an important issue particularly due to the difficult and often incomplete cleaning of the "elevator function" of this scope. The incidence of endoscope-related infectious transmission has been reported to be 3.7 and 1.6 per 1,000 gastrointestinal endoscopic procedures [1, 2]. Moreover, it estimated the cost of post-endoscopic infection is nearly $\$ 12,574.28$ per hospitalization in the US, leading to a huge health insurance burden[13]. The cost of reusable endoscopes may be between $\$ 101.16$ and $\$ 238.71$ per endoscopic procedure including purchase, maintenance, reprocessing, and repair [13, 14]. Furthermore, even with strict compliance to all reprocessing and maintenance instructions, such as cleaning, inspection, and repair of the reusable endoscope, device-related infections persist [15].

In the present study, we demonstrated that the use of a novel disposable endoscope was as feasible and safe as the conventional endoscope for upper gastrointestinal tract examination. Moreover, there were several advantages of the novel disposable endoscope. First, the novel disposable endoscope may be more effective in preventing endoscope-related infections. Second, the novel disposable endoscope may be cost-effective in terms of reprocessing, personnel, maintenance, and repair cost. The cost of the novel disposable endoscope may be between $\$ 100$ and $\$ 150$ in the future. However, the estimated cost of reusable endoscopes is between $\$ 101.16$ and $\$ 238.71$ per examination $[13,14]$. Therefore, the cost of the novel disposable endoscope may be relatively lower compared with the conventional endoscope once such an approach becomes widely utilized. Third, time spent on reprocessing will be saved in high-volume endoscopic procedure centers once the novel disposable endoscope becomes more widely used. Although the procedure duration of the novel disposable endoscope was longer compared with the conventional endoscope, this could potentially ameliorate with a growing expertise. Fourth, the disposable endoscope may also be beneficial to patients living in remote areas that do not have stringent reprocessing facilities.

Our study has some limitations. First, the sample size in this study was relatively small, and the feasibility and safety study of the novel disposable endoscope must be assessed further in larger cohorts. Second, the patient's comfort level with the novel disposable endoscope could not be assessed as all patients were sedated during the procedure. Third, the effect of intra-observer and inter-observer bias could not be removed despite all endoscopists being adequately trained before the procedure. Fourth, the novel disposable endoscope was not capable of performing electronic staining and magnification, which may restrict the early screening of upper gastrointestinal tract cancer. However, as no premalignant or malignant lesions were detected in either group, it was difficult to assess whether the novel disposable endoscope was as good as the conventional endoscope in detecting premalignant or malignant lesions. Fifth, it is still unknown whether the novel disposable endoscope can be used for therapeutic procedures. Indeed, this would be a very important quality measure in the fu- 
ture. Sixth, the procedure duration of the novel disposable endoscope was significantly longer compared to the conventional endoscope. Seventh, as erosive esophagitis was an exclusion criterion in this study, this limited our ability to assess if the disposable endoscope lacerated the esophagus.

Collectively, the novel disposable endoscope was as feasible and safe as the conventional endoscope for upper gastrointestinal tract examination. However, further studies are required to confirm whether the novel disposable endoscope can be widely used in clinical practice.

\section{Author contributions}

Li-sheng Wang and Jun Yao were responsible for the design of the study and reviewed the manuscript. Tao Dong and Yong-de Cai trained for the use of the novel disposable upper gastrointestinal endoscope. De-Feng Li performed data analysis and drafted the manuscript. Rui-yue Shi and Yan-hui Tian recorded data. Li-sheng Wang, Zheng-lei Xu, Ying-sheng Zhou, Xian-jiu Sun, Jin-wei Cai, Yuan-yuan Fang, Huan Peng, and Jia-min Wang performed the procedure. Jun Yao and Li-sheng Wang were responsible for revising the manuscript. All authors have read and approved the final manuscript.

Funding

Natural Science Foundation of Guangdong Province | http://dx.doi.org/ 10.13039/501100003453 (No.2018A0303100024) I Technical Research and Development Project of Shenzhen (No. JCYJ20150403101028164, No. JCYC201703071009114,No. JCYJ20190807145617113) | Shenzhen Health Planning Commission (Shenzhen Health Planning Commission) | National Natural Science Foundation of China | http://dx.doi.org/10.13039/501100001809 (No.81502040) | Three Engineering Training Funds in Shenzhen (No. SYLY201718, No.SYJY201714 and No.SYLY201801)

\section{Conflict of Interest}

The authors declare that they have no conflict of interest.

\section{Acknowledgement}

This work was supported by the Natural Science Foundation of Guangdong Province (No.2018A0303100024), Three Engineering Training Funds in Shenzhen (No. SYLY201718, No. SYJY201714, and No. SYLY201801), Technical Research and Development Project of Shenzhen (No. JCYJ20150403101028164, No. JCYC20170307100911479, and No. JCYJ20190807145617113), National Natural Science Foundation of China (No.81502040), and Shenzhen Health Planning Commission (No. SZXJ2017030).

\section{References}

[1] Wang P, Xu T, Ngamruengphong S et al. Rates of infection after colonoscopy and osophagogastroduodenoscopy in ambulatory surgery centres in the USA. Gut 2018; 67: 1626-1636. doi:10.1136/gutjnl-2017315308

[2] Lin JN, Wang CB, Yang CH et al. Risk of infection following colonoscopy and sigmoidoscopy in symptomatic patients. Endoscopy 2017; 49: 754764. doi:10.1055/s-0043-107777

[3] Bisset L, Cossart YE, Selby W et al. A prospective study of the efficacy of routine decontamination for gastrointestinal endoscopes and the risk factors for failure. Am J Infect Control 2006; 34: 274-280. doi:10.1016/j. ajic.2005.08.007

[4] Ross AS, Baliga C, Verma P et al. A quarantine process for the resolution of duodenoscope-associated transmission of multidrug-resistant Escherichia coli. Gastrointest Endosc 2015; 82: 477-483. doi:10.1016/j. gie.2015.04.036

[5] Guan W], Ni ZY, Hu Y et al. Clinical characteristics of coronavirus disease 2019 in China. N Engl J Med 2020; 382: 1708-1720. doi:10.1056/NEJMoa2002032

[6] Zhou M, Zhang X, Qu J. Coronavirus disease 2019 (COVID-19): a clinical update. Front Med 2020; 14: 126-135. doi:10.1007/s11684-020-07678

[7] Tan C, Xiao Y, Wu Y. Gastrointestinal endoscopy operation-a potential transmission risk for SARS-CoV-2. Am J Infect Control 2020; 44: 11251126. doi:10.1016/j.ajic.2020.05.025

[8] Siddique SM, Sultan S, Lim JK et al. Spotlight: COVID-19 PPE and endoscopy. Gastroenterology 2020; 159: 759. doi:10.1053/j.gastro.2020.06.047

[9] Folch E, Kheir F, Mahajan A et al. Bronchoscope-guided percutaneous endoscopic gastrostomy tube placement by interventional pulmonologists: a feasibility and safety study. J Intensive Care Med 2018; 885066618800275: doi:10.1177/0885066618800275

[10] Muthusamy VR, Bruno M], Kozarek RA et al. Clinical evaluation of a single-use duodenoscope for endoscopic retrograde cholangiopancreatography. Clin Gastroenterol Hepatol 2019; 18: 2108-2117. doi:10.1016/ j.cgh.2019.10.052

[11] O'Horo JC, Farrell A, Sohail MR, Safdar N. Carbapenem-resistant Enterobacteriaceae and endoscopy: an evolving threat. Am J Infect Control 2016; 44: 1032-1036. doi:10.1016/j.ajic.2016.03.029

[12] Naas T, Cuzon G, Babics A et al. Endoscopy-associated transmission of carbapenem-resistant Klebsiella pneumoniae producing KPC-2 betalactamase. J Antimicrob Chemother 2010; 65: 1305-1306. doi:10.1093/ $\mathrm{jac} / \mathrm{dkq} 117$

[13] Larsen S, Kalloo A, Hutfless S. The hidden cost of colonoscopy including cost of reprocessing and infection rate: the implications for disposable colonoscopes. Gut 2020; 69: 197-200. doi:10.1136/gutjnl-2019319108

[14] Bang JY, Sutton B, Hawes R, Varadarajulu S. Concept of disposable duodenoscope: at what cost? Gut 2019; 68: 1915-1917. doi:10.1136/ gutjnl-2019-318227

[15] McCafferty CE, Aghajani MJ, Abi-Hanna D et al. An update on gastrointestinal endoscopy-associated infections and their contributing factors. Ann Clin Microbiol Antimicrob 2018; 17: 36. doi:10.1186/s12941-0180289-2 